\title{
Lone Pair Electron Discriminate Hybridization with Aromatic and Anti Aromatic behavior of Heterocyclic Compounds - Innovative Mnemonics
}

\author{
Arijit Das $^{*}$ \\ Department of Chemistry, Ramthakur College, Agartala -799003, Tripura West, Tripura, India \\ *Corresponding author: arijitdas78chem@gmail.com, arijitdas_chem@rediffmail.com
}

\begin{abstract}
In this approach, formulae based mnemonics by counting lone pair of electrons (localized or delocalized) have been highlighted by innovative and time economic way to enhance interest of students' who belong to paranoia zone of chemistry for the prediction of Hybridization state of hetero atom and Aromatic, Anti aromatic, non aromatic behavior of different heterocyclic compounds. Here, I have tried to hub three (03) time economic mnemonics by including three (03) formulae for the prediction of hybridization of hetero atom, aromatic and anti aromatic behavior of heterocyclic compounds. This article encourages students to solve multiple choice type questions (MCQs) on 'Aromaticity of Heterocyclic compounds' at different competitive examinations in a time economic ground.
\end{abstract}

Keywords: high school, under graduate student, graduate student, post graduate student, chemical education research, heterocyclic compound, DLP, LLP, hybridization, aromatic, anti aromatic and non aromatic

Cite This Article: Arijit Das, "Lone Pair Electron Discriminate Hybridization with Aromatic and Anti Aromatic behavior of Heterocyclic Compounds - Innovative Mnemonics." World Journal of Chemical Education, vol. 6, no. 2 (2018): 95-101. doi: 10.12691/wjce-6-2-4.

\section{Introduction}

The conventional methods [1-7] for determination of hybridization state of hetero atom, prediction of aromatic and anti aromatic nature of heterocyclic compound is time consuming. Keeping this in mind, in this article, I have introduced three time economic innovative mnemonics by using three formulae for the prediction of hybridization state of hetero atom, aromatic and anti aromatic nature of heterocyclic compounds containing one, two or more number of hetero atoms to make heterocyclic chemistry metabolic and interesting for students. This study also shows how LP electrons discriminate prediction of hybridization state of hetero atom with identification of its Aromatic and Anti Aromatic nature of heterocyclic compounds.

\section{Time Economic Innovative Mnemonics in Heterocyclic Chemistry}

\subsection{Classification of Lone Pair of Electrons}

Loan Pair of electrons can be generally classified into two types as Delocalized lone pair of electron (DLP) and Localized lone pair of electron (LLP) as follows:

i)Delocalized lone pair of electron (DLP): When lone pair of electron of hetero atom undergo delocalization through conjugation then it is to be treated as delocalized lone pair of electron (DLP). Hetero atom (atom containing lone pair of electron) which is directly attached with single bonds only from all ends is to be considered as DLP containing hetero atom and its lone pair is to be treated as DLP.<smiles>c1ccncc1</smiles>

Eg. In Pyrrole $\mathrm{H}$ lone pair of $\mathrm{N}$ atom is to be treated as DLP because it is directly attached with three single bonds only.

ii) Localized lone pair of electron (LLP): When lone pair of electron of hetero atom does not undergo delocalization through conjugation then it is to be treated as Localized lone pair of electron (LLP). Hetero atom (atom containing lone pair of electron) which is directly attached with single and double bonds with the ring system is to be considered as LLP containing hetero atom and its lone pair is to be treated as localized lone pair of electron (LLP).<smiles>c1ccncc1</smiles>

Eg. In Pyridine $\quad \cdots \quad$ lone pair of $\mathrm{N}$ atom is to be treated as LLP because it is directly attached with double and single bonds with the ring system. 


\subsection{Hybridization State Theory}

Prof. Linus Pauling (1931) first developed the Hybridization state theory in order to explain the structure of molecules such as methane $\left(\mathrm{CH}_{4}\right)$. This concept was developed for simple chemical systems but this one applied more widely later on and from today's point of view it is considered an operative empirical for excusing the structures of organic and inorganic compounds along with their related problems.

Conventional method for prediction of hybridization state:

Hybridization state for a molecule can be calculated by the formula $0.5(\mathrm{~V}+\mathrm{H}-\mathrm{C}+\mathrm{A})$, Where, $\mathrm{V}=$ Number of valance electrons in central atom, $H=$ Number of surrounding monovalent atoms, $\mathrm{C}=$ Cationic charge, $\mathrm{A}=$ Anionic charge.
Innovative Mnemonics for the prediction of hybridization state of hetero atom in the heterocyclic compounds with LLP:

Formula used for the determination of hybridization state:

Power on the Hybridization state of the hetero atom $=$ (Total no of $\sigma$ bonds around each hetero atom - 1).

This formula should be applicable up to $4 \sigma$ bonds.

If the power of the hybridization state will be 03,02 and 01 then the hybridization state will be $\mathrm{sp}^{3}, \mathrm{sp}^{2}$ and $\mathrm{sp}$ respectively. All single (-) bonds are $\sigma$ bond, in double bond $(=)$ there is one $\sigma$ and one $\pi$. In addition to these each localized lone pair of electron (LLP) can be treated as one $\sigma$ bond. Hybridization state of hetero atom with the help of LLP in heterocyclic compounds are shown in Table 1 below.

Table 1. (Hybridization state of Hetero atom in Heterocyclic Compounds with the help of LLP)

\begin{tabular}{|c|c|c|c|c|}
\hline Heterocyclic Compounds & $\begin{array}{l}\text { Number of } \sigma \text { bonds } \\
\text { around hetero atom } \\
\text { (from single and double } \\
\text { bonds) } \\
\text { (A) }\end{array}$ & $\begin{array}{c}\text { LLP } \\
\left.\text { (localized Lone Pair of } \mathrm{e}^{-} \mathrm{s}\right) \\
\text { (B) }\end{array}$ & $\begin{array}{l}\text { Total Number of } \sigma \text { bonds } \\
\text { around hetero atom } \\
(\mathrm{A}+\mathrm{B})\end{array}$ & $\begin{array}{c}\text { Power of the } \\
\text { Hybridization state } \\
\text { of the hetero atom } \\
\text { (Corresponding } \\
\text { Hybridization state) }= \\
(\mathrm{A}+\mathrm{B})-1\end{array}$ \\
\hline $\begin{array}{c}\mathrm{H} \\
\text { Pyrrole } \\
\end{array}$ & 03 & $\begin{array}{c}0 \\
\text { (lone pair of electron } \\
\text { undergo delocalization,DLP } \\
\text { with the ring system) }\end{array}$ & 03 & $\begin{array}{c}02 \\
\left(\mathrm{sp}^{2} \mathrm{~N}\right)\end{array}$ \\
\hline $\begin{array}{c}\mathrm{O}^{\prime} \\
\text { •• } \\
\text { Furan }\end{array}$ & 02 & $\begin{array}{c}01 \\
\text { (out of two lone pair of } \\
\text { electrons, one undergo } \\
\text { delocalization,DLP and other } \\
\text { remain as LLP) }\end{array}$ & 03 & $\begin{array}{c}02 \\
\left(\mathrm{sp}^{2} \mathrm{O}\right)\end{array}$ \\
\hline$\stackrel{\ddot{\mathrm{S}}}{\bullet}$ & 02 & $\begin{array}{c}01 \\
\text { (out of two lone pair of } \\
\text { electrons, one undergo } \\
\text { delocalization,DLP and other } \\
\text { remain as LLP) }\end{array}$ & 03 & $\begin{array}{c}02 \\
\left(\mathrm{sp}^{2} \mathrm{~S}\right)\end{array}$ \\
\hline Pyridine & 02 & 01 & 03 & $\begin{array}{c}02 \\
\left(\mathrm{sp}^{2} \mathrm{~N}\right)\end{array}$ \\
\hline Indole & 03 & 0 & 03 & $\begin{array}{c}02 \\
\left(\mathrm{sp}^{2} \mathrm{~N}\right)\end{array}$ \\
\hline Quinoline & 02 & 01 & 03 & $\begin{array}{c}02 \\
\left(\mathrm{sp}^{2} \mathrm{~N}\right)\end{array}$ \\
\hline Isoquinoline & 02 & 01 & 03 & $\begin{array}{c}02 \\
\left(\mathrm{sp}^{2} \mathrm{~N}\right)\end{array}$ \\
\hline
\end{tabular}




\begin{tabular}{|c|c|c|c|c|}
\hline Heterocyclic Compounds & $\begin{array}{l}\text { Number of } \sigma \text { bonds } \\
\text { around hetero atom } \\
\text { (from single and double } \\
\text { bonds) } \\
\text { (A) }\end{array}$ & $\begin{array}{l}\text { LLP } \\
\text { (localized Lone Pair of e`s) } \\
\text { (B) }\end{array}$ & $\begin{array}{c}\text { Total Number of } \sigma \text { bonds } \\
\text { around hetero atom } \\
(\mathrm{A}+\mathrm{B})\end{array}$ & $\begin{array}{c}\text { Power of the } \\
\text { Hybridization state } \\
\text { of the hetero atom } \\
\text { (Corresponding } \\
\text { Hybridization state) }= \\
\text { (A+B)-1 }\end{array}$ \\
\hline$\left.\left.\right|_{\substack{\mathrm{H} \\
\text { Imidazole }}} ^{3}\right|_{5} ^{3}$ & $\begin{array}{c}03 \\
(\mathrm{~N} 1) \\
02 \\
(\mathrm{~N} 3)\end{array}$ & $\begin{array}{c}0 \\
(\mathrm{~N} 1) \\
01 \\
(\mathrm{~N} 3)\end{array}$ & $\begin{array}{l}03 \\
03\end{array}$ & $\begin{array}{c}02 \\
\left(\mathrm{sp}^{2} \mathrm{~N} 1\right) \\
\\
02 \\
\left(\mathrm{sp}^{2} \mathrm{~N} 3\right)\end{array}$ \\
\hline Pyrimidine & $\begin{array}{c}02 \\
(\mathrm{~N} 1) \\
\\
02 \\
(\mathrm{~N} 3)\end{array}$ & $\begin{array}{c}01 \\
(\mathrm{~N} 1) \\
\\
01 \\
\text { (N3) }\end{array}$ & $\begin{array}{l}03 \\
03\end{array}$ & $\begin{array}{c}02 \\
\left(\mathrm{sp}^{2} \mathrm{~N} 1\right) \\
\\
02 \\
\left(\mathrm{sp}^{2} \mathrm{~N} 3\right)\end{array}$ \\
\hline$={ }_{3}^{3}$ & $\begin{array}{c}02 \\
(\mathrm{~N} 1) \\
02 \\
(\mathrm{~N} 3) \\
02 \\
\text { (N7) } \\
03 \\
\text { (N9) } \\
\end{array}$ & $\begin{array}{c}01 \\
(\mathrm{~N} 1) \\
01 \\
(\mathrm{~N} 3) \\
01 \\
(\mathrm{~N} 7) \\
0 \\
(\mathrm{~N} 9) \\
\end{array}$ & $\begin{array}{l}03 \\
03 \\
03 \\
03 \\
03\end{array}$ & $\begin{array}{c}02 \\
\left(\mathrm{sp}^{2} \mathrm{~N} 1\right) \\
02 \\
02 \\
\left(\mathrm{sp}^{2} \mathrm{~N} 3\right) \\
02 \\
\left(\mathrm{sp}^{2} \mathrm{~N} 7\right) \\
02 \\
02 \\
\left(\mathrm{sp}^{2} \mathrm{~N} 9\right)\end{array}$ \\
\hline $\begin{array}{c}\stackrel{S}{\ddot{0}} \\
\text { Thiazole }\end{array}$ & $\begin{array}{l}02 \\
(\mathrm{~N}) \\
\\
02 \\
(\mathrm{~S})\end{array}$ & $\begin{array}{c}01 \\
(\mathrm{~N}) \\
01 \\
(\mathrm{~S}) \\
\text { (out of two lone pair of } \\
\text { electrons on S, one undergo } \\
\text { delocalization, DLP and } \\
\text { other remain as LLP) }\end{array}$ & $\begin{array}{l}03 \\
03\end{array}$ & $\begin{array}{c}02 \\
\left(\mathrm{sp}^{2} \mathrm{~N}\right) \\
\\
02 \\
\left(\mathrm{sp}^{2} \mathrm{~S}\right)\end{array}$ \\
\hline $\begin{array}{c}\text { Ș }^{\prime} \\
\text { Benzothiazole }\end{array}$ & $\begin{array}{l}02 \\
(\mathrm{~N}) \\
\\
02 \\
(\mathrm{~S})\end{array}$ & $\begin{array}{c}01 \\
(\mathrm{~N}) \\
01 \\
(\mathrm{~S}) \\
\text { (out of two lone pair of } \\
\text { electrons on S, one undergo } \\
\text { delocalization, DLP and } \\
\text { other remain as LLP) } \\
\end{array}$ & $\begin{array}{l}03 \\
03\end{array}$ & $\begin{array}{c}02 \\
\left(\mathrm{sp}^{2} \mathrm{~N}\right) \\
\\
02 \\
\left(\mathrm{sp}^{2} \mathrm{~S}\right)\end{array}$ \\
\hline $\begin{array}{c}2 \\
1 \\
1 \\
\text { Pyrazine } \\
\text { (p-diazine) }\end{array}$ & $\begin{array}{c}02 \\
(\mathrm{~N} 1) \\
\\
02 \\
(\mathrm{~N} 1)\end{array}$ & $\begin{array}{c}01 \\
(\mathrm{~N} 1) \\
\\
01 \\
\text { (N1) }\end{array}$ & $\begin{array}{l}03 \\
03\end{array}$ & $\begin{array}{c}02 \\
\left(\mathrm{sp}^{2} \mathrm{~N} 1\right) \\
\\
02 \\
\left(\mathrm{sp}^{2} \mathrm{~N} 4\right)\end{array}$ \\
\hline$N_{\text {Cyanidine }}^{N}$ & $\begin{array}{c}02 \\
(\mathrm{~N} 1, \mathrm{~N} 3 \text { and N5) }\end{array}$ & $\begin{array}{c}01 \\
(\mathrm{~N} 1, \mathrm{~N} 3 \text { and N5) }\end{array}$ & 03 & $\begin{array}{c}02 \\
\left(\mathrm{sp}^{2} \mathrm{~N} 1, \mathrm{~N} 3, \mathrm{~N} 5\right)\end{array}$ \\
\hline $\begin{array}{c}\ddot{S} \\
\text { Phenothiazine }\end{array}$ & $\begin{array}{l}03 \\
(\mathrm{~N}) \\
02 \\
(\mathrm{~S})\end{array}$ & $\begin{array}{c}0 \\
(\mathrm{~N}) \\
01 \\
(\mathrm{~S}) \\
\text { (out of two lone pair of } \\
\text { electrons on S, one undergo } \\
\text { delocalization, DLP and } \\
\text { other remain as LLP) }\end{array}$ & $\begin{array}{l}03 \\
03\end{array}$ & $\begin{array}{c}02 \\
\left(\mathrm{sp}^{2} \mathrm{~N}\right) \\
\\
02 \\
\left(\mathrm{sp}^{2} \mathrm{~S}\right)\end{array}$ \\
\hline
\end{tabular}




\begin{tabular}{|c|c|c|c|c|}
\hline Heterocyclic Compounds & $\begin{array}{l}\text { Number of } \sigma \text { bonds } \\
\text { around hetero atom } \\
\text { (from single and double } \\
\text { bonds) } \\
\text { (A) }\end{array}$ & $\begin{array}{l}\text { LLP } \\
\left.\text { (localized Lone Pair of e } \mathrm{e}^{-\mathrm{s}}\right) \\
\text { (B) }\end{array}$ & $\begin{array}{l}\text { Total Number of } \sigma \text { bonds } \\
\text { around hetero atom } \\
(\mathrm{A}+\mathrm{B})\end{array}$ & $\begin{array}{c}\text { Power of the } \\
\text { Hybridization state } \\
\text { of the hetero atom } \\
\text { (Corresponding } \\
\text { Hybridization state) }= \\
\text { (A+B)-1 }\end{array}$ \\
\hline Phenazine & $\begin{array}{c}02 \\
\text { (both } \mathrm{N})\end{array}$ & $\begin{array}{c}01 \\
(\text { both } \mathrm{N})\end{array}$ & 03 & $\begin{array}{c}02 \\
\left(\mathrm{sp}^{2} \text { both } \mathrm{N}\right)\end{array}$ \\
\hline 1,2,3,4-tetrazine & $\begin{array}{c}02 \\
(\mathrm{~N} 1, \mathrm{~N} 2, \mathrm{~N} 3, \mathrm{~N} 4)\end{array}$ & $\begin{array}{c}01 \\
(\mathrm{~N} 1, \mathrm{~N} 2, \mathrm{~N} 3, \mathrm{~N} 4)\end{array}$ & 03 & $\begin{array}{c}02 \\
\left(\mathrm{sp}^{2} \mathrm{All} \mathrm{N}\right)\end{array}$ \\
\hline Azocine & 02 & 01 & 03 & $\begin{array}{c}02 \\
\left(\mathrm{sp}^{2} \mathrm{~N}\right)\end{array}$ \\
\hline $\begin{array}{l}\mathrm{N}: \\
\text { Azetine }\end{array}$ & 02 & 01 & 03 & $\begin{array}{c}02 \\
\left(\mathrm{sp}^{2} \mathrm{~N}\right)\end{array}$ \\
\hline$\sum_{\text {Aziridine }}^{\mathrm{H}}$ & 03 & 01 & 04 & $\begin{array}{c}03 \\
\left(\mathrm{sp}^{3} \mathrm{~N}\right)\end{array}$ \\
\hline$\ddot{O}:$ & 02 & 02 & 04 & $\begin{array}{c}03 \\
\left(\mathrm{sp}^{3} \mathrm{O}\right)\end{array}$ \\
\hline
\end{tabular}

\subsection{Aromaticity}

It was first devised by Hückel in 1931.

Conventional method for prediction of Aromatic nature of organic compound:

1. Cyclic molecule,

2. Planer molecule in which all bonded atoms lie in same plane (having $\mathrm{sp}^{2}$ hybridized)

3. Conjugated molecule with conjugated $\pi$-electron system,

4. Contains $(4 n+2) \pi$ electrons, where, $n$ is a positive integer $(\mathrm{n}=0,1,2,3$ etc. $)$

Conventional method for Anti Aromatic nature of organic Compound:

1. Cyclic molecule,

2. Planer molecule in which all bonded atoms lie in same plane (having $\mathrm{sp}^{2}$ hybridized)

3. Conjugated molecule with conjugated $\pi$-electron system,

4. $4 \mathrm{n} \pi$ electrons, where, $\mathrm{n}$ is a positive integer $(\mathrm{n}=0,1,2,3$ etc. $)$

Conventional method for identification of Non Aromatic Nature of organic Compound:

If a compound violates any one of the above three conditions ( 1 or 2 or 3 ) then it is non aromatic in nature.

Innovative Mnemonics for the Prediction of Aromatic, Anti Aromatic behavior of Heterocyclic Compounds with DLP:
The present study will be an innovative mnemonic involving calculation of ' $A$ ' value by just manipulating the no of $\pi$ bonds within the ring system and delocalized lone pair of electron (DLP) with one (01).

The heterocyclic compound having cyclic, planar, conjugated (i.e. all the carbon atoms having same state of hybridization, $\mathrm{sp}^{2}$ ) with even number of ' $\mathrm{A}$ ' value will be treated as aromatic in nature and with odd number of ' $A$ ' value will be treated as anti aromatic in nature.

\subsection{Evaluation of A Value}

$$
\begin{aligned}
\mathbf{A} & =\pi \mathbf{b}+\mathbf{D L P}+\mathbf{1}(\text { constant }) \\
& =\text { even no }=\text { Aromatic } \\
\mathbf{A} & =\pi \mathbf{b}+\mathbf{D L P}+\mathbf{1}(\text { constant }) \\
& =\text { odd } \mathbf{n o}=\text { Anti Aromatic }
\end{aligned}
$$

where, $\pi b=$ number of $\pi$ bonds with in the ring system; DLP $=$ Delocalized lone pair of electron.

In case of a multi hetero atom based heterocyclic compound, containing both DLP and LLP hetero atoms, Aromatic and Anti Aromatic behaviour should be predicted with respect to DLP based hetero atom only.

But when both the hetero atoms are in the same mode of classification (both DLP or both LLP) then Aromaticity should be predicted with respect to that hetero atom which contains lowest possible position number as per IUPAC nomenclature. 
Eg. Imidazole is a multi hetero atom based hetero cyclic compound in which, N1 is DLP based hetero atom and N3 is LLP based hetero atom. In this case Aromaticity should be predicted with respect to the DLP based hetero atom N1.

For $\mathrm{N} 1, \mathrm{~A}=\pi \mathrm{b}+\mathrm{DLP}+1($ constant $)=2+1+1=4($ even no) - Aromatic

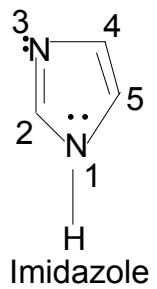

Eg. Pyrimidine is a multi hetero atom based hetero cyclic compound in which, both N1 \& N3 are in same environment based hetero atoms (LLP based hetero atoms). In this case Aromaticity should be predicted with respect to N1 (lowest possible position number as per IUPAC nemenclature).

For $\mathrm{N} 1, \mathrm{~A}=\pi \mathrm{b}+\mathrm{DLP}+1($ constant $)=3+0+1=4($ even no) - Aromatic

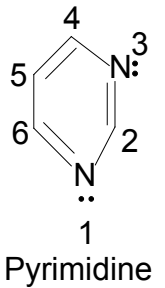

Aromaticity of heterocyclic compounds have been illustrated in Table 2.

Table 2. (Aromatic-Anti Aromatic and Non Aromatic behavior of heterocyclic compounds With the help of DLP)

\begin{tabular}{|c|c|c|c|c|}
\hline $\begin{array}{l}\text { Hetero Cyclic Compound } \\
\text { (Cyclic, Planar, Conjugated) }\end{array}$ & $\begin{array}{c}\pi \mathrm{b} \text { value } \\
{[\pi \mathrm{b}=\text { number of } \pi} \\
\text { bonds with in the ring } \\
\text { system }]\end{array}$ & DLP & $\begin{array}{c}\text { A value } \\
{[\mathrm{A}=\pi \mathrm{b}+\mathrm{DLP}+1(\text { constant })]} \\
(\text { even no/odd no })\end{array}$ & $\begin{array}{c}\text { Remark on Nature of } \\
\text { compound (Aromatic/Anti } \\
\text { Aromatic) }\end{array}$ \\
\hline $\begin{array}{c}\mathrm{H} \\
\text { Pyrrole } \\
\end{array}$ & 2 & 1 & $\begin{array}{l}2+1+1=4 \\
\quad(\text { even no })\end{array}$ & Aromatic \\
\hline$\stackrel{\bullet}{\text { Furan }}$ & 2 & $\begin{array}{c}1 \\
\text { ( Here out of two lone } \\
\text { pairs on O only one } \\
\text { LP take part in } \\
\text { delocalization) }\end{array}$ & $\begin{array}{c}2+1+1=4 \\
(\text { even no) }\end{array}$ & Aromatic \\
\hline 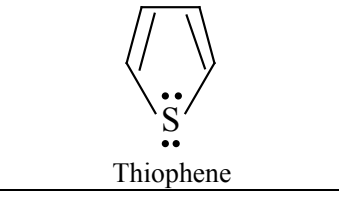 & 2 & $\begin{array}{c}1 \\
\text { (Here out of two lone } \\
\text { pairs on O only one } \\
\text { LP take part in } \\
\text { delocalization) }\end{array}$ & $\begin{array}{l}2+1+1=4 \\
\quad(\text { even no })\end{array}$ & Aromatic \\
\hline & 3 & 0 & $\begin{array}{c}3+0+1=4 \\
(\text { even no) }\end{array}$ & Aromatic \\
\hline Indole & 4 & 1 & $\begin{array}{c}4+1+1=6 \\
(\text { even no })\end{array}$ & Aromatic \\
\hline Quinoline & 5 & 0 & $\begin{array}{c}5+0+1=6 \\
(\text { even no })\end{array}$ & Aromatic \\
\hline Isoquinoline & 05 & 0 & $\begin{array}{c}5+0+1=6 \\
(\text { even no })\end{array}$ & Aromatic \\
\hline
\end{tabular}




\begin{tabular}{|c|c|c|c|c|}
\hline $\begin{array}{l}\text { Hetero Cyclic Compound } \\
\text { (Cyclic, Planar, Conjugated) }\end{array}$ & $\begin{array}{c}\pi \mathrm{b} \text { value } \\
{[\pi \mathrm{b}=\text { number of } \pi} \\
\text { bonds with in the ring } \\
\text { system }]\end{array}$ & DLP & $\begin{array}{c}\text { A value } \\
{[\mathrm{A}=\pi \mathrm{b}+\mathrm{DLP}+1(\text { constant })]} \\
(\text { even no/odd no })\end{array}$ & $\begin{array}{c}\text { Remark on Nature of } \\
\text { compound (Aromatic/Anti } \\
\text { Aromatic) }\end{array}$ \\
\hline$\left.\right|_{\text {Imidazole }} ^{\mathrm{H}}$ & 02 & $\begin{array}{c} \\
01 \\
(\mathrm{~N} 1)\end{array}$ & $\begin{array}{l}2+1+1=4 \\
\quad(\text { even no) }\end{array}$ & Aromatic \\
\hline Pyrimidine & 03 & $\begin{array}{c}0 \\
(\mathrm{~N} 1)\end{array}$ & $\begin{array}{l}3+0+1=4 \\
\quad(\text { even no) }\end{array}$ & Aromatic \\
\hline $\begin{array}{ll}\mathrm{N}^{\prime} & \left.\right|_{9} ^{\mathrm{N}} \\
\text { Purine } & \mathrm{N}\end{array}$ & 04 & $\begin{array}{c}01 \\
(\mathrm{~N} 9)\end{array}$ & $\begin{array}{l}4+1+1=6 \\
\text { (even no) }\end{array}$ & Aromatic \\
\hline $\begin{array}{c}\text { S. } \\
\text { Thiazole }\end{array}$ & 02 & $\begin{array}{l}01 \\
(\mathrm{~S})\end{array}$ & $\begin{array}{l}2+1+1=4 \\
\quad(\text { even no) }\end{array}$ & Aromatic \\
\hline Benzothiazole & 04 & $\begin{array}{l}01 \\
(\mathrm{~S})\end{array}$ & $\begin{array}{l}4+1+1=6 \\
\quad(\text { even no) }\end{array}$ & Aromatic \\
\hline $\begin{array}{c}6 \\
\dddot{1} \\
\text { Pyrazine } \\
\text { (p-diazine) }\end{array}$ & 03 & 0 & $\begin{array}{l}3+0+1=4 \\
\quad(\text { even no) }\end{array}$ & Aromatic \\
\hline Cyanidine & 03 & 0 & $\begin{array}{l}3+0+1=4 \\
\quad(\text { even no) }\end{array}$ & Aromatic \\
\hline Phenothiazine & 06 & $\begin{array}{c}01 \\
(\mathrm{~N}) \\
01 \\
(\mathrm{~S}) \\
\text { (Here out of two lone } \\
\text { pairs on S only one LP } \\
\text { take part in } \\
\text { delocalization) } \\
\end{array}$ & $\begin{array}{l}6+1+1=8 \\
\quad(\text { even no) }\end{array}$ & Aromatic \\
\hline Phenazine & 07 & 0 & $\begin{array}{l}7+0+1=8 \\
\quad(\text { even no) }\end{array}$ & Aromatic \\
\hline
\end{tabular}




\begin{tabular}{|c|c|c|c|c|}
\hline $\begin{array}{l}\text { Hetero Cyclic Compound } \\
\text { (Cyclic, Planar, Conjugated) }\end{array}$ & $\begin{array}{c}\pi \mathrm{b} \text { value } \\
{[\pi \mathrm{b}=\text { number of } \pi} \\
\text { bonds with in the ring } \\
\text { system }]\end{array}$ & DLP & $\begin{array}{c}\text { A value } \\
{[\mathrm{A}=\pi \mathrm{b}+\mathrm{DLP}+1(\text { constant })]} \\
(\text { even no/odd no })\end{array}$ & $\begin{array}{c}\text { Remark on Nature of } \\
\text { compound (Aromatic/Anti } \\
\text { Aromatic) }\end{array}$ \\
\hline 1,2,3,4-tetrazine & 03 & 0 & $\begin{array}{l}3+0+1=4 \\
(\text { even no })\end{array}$ & Aromatic \\
\hline Azocine & 04 & 0 & $\begin{array}{l}4+0+1=5 \\
\quad(\text { odd no) }\end{array}$ & Anti aromatic \\
\hline Azetine & 02 & 0 & $\begin{array}{l}2+0+1=3 \\
\quad(\text { odd no) }\end{array}$ & Anti aromatic \\
\hline$\sum_{\text {Aziridine }}^{\mathrm{H}} \stackrel{\mathrm{N}}{\cdots}$ & - & - & - & $\begin{array}{l}\text { Non Aromatic (non planar- } \\
\qquad \mathrm{sp}^{3} \text { ) }\end{array}$ \\
\hline${ }_{\text {Oxetan }}$ & - & - & - & $\begin{array}{l}\text { Non Aromatic (non planar- } \\
\qquad \mathrm{sp}^{3} \text { ) }\end{array}$ \\
\hline
\end{tabular}

\section{Conclusions}

It may be expected that these three time economic innovative mnemonics will help the student of chemical education at Undergraduate, Senior Undergraduate and Post-Graduate level to predict hybridization state of hetero atom, aromatic and anti aromatic behaviour of heterocyclic compounds. Experiment in vitro on 100 students showed that by using these three formulae students can save up to 5-10 minutes time in the examination hall to find out the aromatic/anti aromatic character and hybridization state of hetero atom for heterocyclic compounds and their comparative study with respect to the classification of lone pair of electron. On the basis of this, I can strongly recommend to use these three time economic innovative mnemonics including three formulae in the field of heterocyclic chemistry.

\section{Acknowledgements}

I am grateful to the SERB, DST, New Delhi, Govt. of India, for their financial assistance (Sanction no - SERB / F / 5537 / 2013-14 dated 27/11/2013 and D.O. No. SB / EMEQ - 014 / 2013).

In my $41^{\text {th }}$ birthday $(14 / 03 / 2018)$, I want to delegate my this innovative research article in the field of heterocyclic chemistry to my beloved father Late Anil Ranjan Das, who was also a chemistry teacher and also founder of my chemistry world (www.arijitchemistryworld.com).
Furthermore, I give my special accolade to Mr. Sanit Debroy, Registrar, Tripura University, India and $\mathrm{Mr}$. Sekhar Datta, Senior Journalist, 'The Telegraph' for their constant valuable sustaining mentality to carry out my innovational research work in the field of chemical education.

In addition to these, finally, I want to give my cordial congratulation to the newly elected Honorable Chief Minister of 'Tripura', Mr.Biplab Kumar Deb and Honorable Education \& Higher Education Minister of Tripura, Mr.Ratan Lal Nath for their constant valuable sustaining mentality to improve our state 'Tripura' in all respect.

\section{References}

[1] L. Pauling, The Nature Chemical Bond. Application of Results Obtained from the Quantum Mechanics and from a Theory of Paramagnetic Susceptibility to the Structure of Molecules, J.Am.Chem.Soc., 1931, 53(4), pp1367-1400.

[2] I.L. Finar, Organic Chemistry, Vol-2, $5^{\text {th }}$ ed., Pearson, 2002, pp606-637, ISBN:81-7808- 164-4.

[3] R.T. Morrison and R.N. Boyd, $6^{\text {th }}$ ed., Organic Chemistry, $6^{\text {th }}$ ed., 1992, pp1059-1061, ISBN: 0-87692-765-7.

[4] T.W.Graham Solomons and C.B. Fryhle, Organic Chemistry, $9^{\text {th }}$ ed., Wiley: India, 2012, pp 655-658, ISBN: 978-81-265-3687-0.

[5] J.G. Smith, Organic Chemistry, $2^{\text {nd }}$ ed., 2008, pp616-619.

[6] Jerry March, Advanced Organic Chemistry: Reaction, Mechanisms and Structure, $4^{\text {th }}$ ed. A Willy-Interscience Publication, JOHN WILEY \& SONS, New York, 2005, p45, ISBN: 9971-51-257-2.

[7] A. Bahl \& B.S. Bahl, A Text Book of Organic Chemistry, $22^{\text {nd }}$ ed., S. Chand \& Company Pvt. Ltd., New Delhi, India, 2016, pp900920, ISBN: 978-93-525-3196-7. 